\title{
Polymeric rapid prototyping for inexpensive and portable medical diagnostics
}

Tianchi Ma, Victoria Northrup, Andrew O. Fung, D. Moira Glerum, Christopher J. Backhouse

Tianchi Ma, Victoria Northrup, Andrew O. Fung, D. Moira Glerum, Christopher J. Backhouse, "Polymeric rapid prototyping for inexpensive and portable medical diagnostics," Proc. SPIE 8412, Photonics North 2012, 84120B (24 October 2012); doi: 10.1117/12.2001470 


\title{
Polymeric rapid prototyping for inexpensive and portable medical diagnostics
}

\author{
Tianchi Ma ${ }^{a b+}$, Victoria Northrup ${ }^{c+}$, Andrew O. Fung ${ }^{a}$, D. Moira Glerum ${ }^{d c b}$ and Christopher \\ J. Backhouse ${ }^{a b^{*}}$ \\ ${ }^{a}$ Dept. of Electrical and Computer Engineering, University of Waterloo, 200 University \\ Avenue West, Waterloo, Ontario N2L 3G1 Canada; \\ ${ }^{b}$ Waterloo Institute of Nanotechnology, University of Waterloo, 200 University Avenue West, \\ Waterloo, Ontario N2L 3G1 Canada; \\ ${ }^{c}$ Dept. of Cell Biology, 5-14 MSB, University of Alberta, Edmonton, Alberta, T6G 2H7 \\ Canada; \\ ${ }^{d}$ Dept. of Biology, University of Waterloo, 200 University Avenue West, Waterloo, Ontario \\ N2L 3G1 Canada; \\ + These authors contributed equally.
}

\begin{abstract}
The advent of inexpensive $\mathrm{CO}_{2}$ laser systems has led to a wide range of demonstrations of microfabricated lab on chip systems built of acrylic. However, there has been little application of these systems to building microfluidics for DNA analysis. In this work we explore the use of $\mathrm{CO}_{2}$ laser systems for building microfluidics for DNA analysis and relate the artifacts of the fabrication technology to the performance of the system. We show that surface roughness that leads to significant constrictions in the separation channel provides an upper limit of the size of DNA that can be analysed. Below that upper limit, the resolution of the chip is strongly affected by the degree to which the separation channel is exposed to redeposited by-products of the ablation process. We show that by controlling these effects we are reliably able to discern two types of PCR product as a test representative of a real application. By being able to do this is in microfluidic devices the size of a postage stamp we have shown that we can now use $\mathrm{CO}_{2}$ laser systems for the development of extremely inexpensive diagnostic systems using a rapid prototyping approach.
\end{abstract}

Keywords: Electrophoresis, Acrylic, PMMA, DNA, separation

\section{INTRODUCTION}

The work of Klank et al. ${ }^{1}$ (2002) effectively introduced the use of $\mathrm{CO}_{2}$ laser systems for the rapid prototyping of microfluidic systems. Since that time, these laser systems have been applied to a wide variety of applications, particularly with poly(methyl methacrylate) (i.e. PMMA). Considerable early development was done of PMMAbased electrophoretic devices for DNA analysis, notably by Caliper (e.g. ${ }^{2}$ ) and Aclara (e.g. ${ }^{3}$ ). More recently we note the very successful demonstrations of embossed and injection moulded PMMA DNA analysis systems, e.g. by Liedert et al. (2012). ${ }^{4}$ With $\mathrm{CO}_{2}$ laser fabricated devices, demonstrations have ranged from PCR systems $^{5}$ to cell culture. This body of work was well-summarised by Zhang and Xing. ${ }^{6}$ However, there has been surprisingly little development of DNA analysis methods on $\mathrm{CO}_{2}$-laser-built microfluidics, with the work of Sun et al. ${ }^{7}$ being one of the few exceptions.

It has been suggested that the ablation process leaves a surface with a charge that is less uniform than that of the pristine surface (e.g. ${ }^{8}$ ), leading to variations in the zeta potential and electro-osmotic flow (EOF). This variation will strongly affect the resolution of the system - a criterion of considerable importance in DNA analysis. It is likely that this is a major barrier to the effective use of $\mathrm{CO}_{2}$-laser-built microfluidics for DNA analysis applications. We decided to explore this by choosing to use agarose as a seiving matrix since agarose, once gelled, will not be as susceptible to EOF variations.

Further author information: (Send correspondence to C.J.B.)

C.J.B.: E-mail: chrisb@uwaterloo.ca, Telephone: +1 (519) 8884567 x31467

Photonics North 2012, edited by Jean-Claude Kieffer, Proc. of SPIE Vol. 8412, 84120B

(C) 2012 SPIE $\cdot$ CCC code: $/ 12 / \$ 18 \cdot$ doi: $10.1117 / 12.2001470$

Proc. of SPIE Vol. $841284120 \mathrm{~B}-1$ 


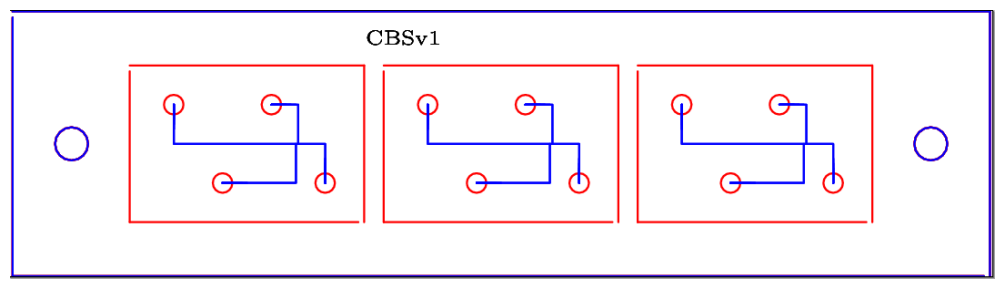

Figure 1. Composite design of 3 small electrophoretic separation chips, each containing two intersecting channels that link 4 wells of approximately $6 \mu \mathrm{L}$ in volume. The chips are held in a larger assembly $10 \mathrm{~cm} \times 2.7 \mathrm{~cm}$ in size, and contain 2 alignment holes. The Well at the top left of each is the buffer waste (BW), that on the top right is the sample well (S), that on the bottom left is the sample waste $(\mathrm{SW})$ and that on the bottom right is the buffer (B) well.

\section{METHODS AND MATERIALS}

This procedure will be described in more detail elsewhere, but, briefly:

\subsection{Materials}

Extruded sheets of 1.5-mm thick Acrylite-FF PMMA were purchased from Evonik Industries (Germany). The agarose was type Agarose III purchased from Amresco Inc. (part CAS-No-9012-36-6). All other materials were purchased from Fisher Scientific unless otherwise noted.

\subsection{Microfabrication}

The design files for this work were created with Asymptote, ${ }^{9}$ a graphics language, and output as postscript files. Unless otherwise specified, the designs were then imported into, and printed from CorelDraw X5 (Corel Corp., version 15.0.0.486) with a windows driver/'Universal Control Panel' with version number 5.31.54.5) as installed from the laser engraver installation media. The laser engraver itself is a $30 \mathrm{~W} 10.6 \mu \mathrm{m} \mathrm{CO}_{2}$ laser system (VLS 2.30) from Universal Laser Systems.

These designs were printed in two sections, each approximately $10 \mathrm{~cm}$ long and containing two alignment holes. The sections also contained the channels and wells such that, once completed, they resembled Fig. 1. The separation and injection channels are approximately 19.6 and $16.7 \mathrm{~mm}$ in length respectively. The distinction between engraving shallow channels $(\approx 105 \pm 5 \mu \mathrm{m})$ in the PMMA and cutting through the PMMA is made by assigning distinct power settings to the colours at each stage. These power settings are summarised in Table 1. The control pannel of the printer easily allows for the sections to be printed in arrays. To increase smoothness, the channels are printed in two passes.

As described nicely by Sun et al., ${ }^{7}$ the PMMA is depolymerized to the monomer at high temperatures and this can readily condense near the region of ablation to form a liquid on the surface (referred to as 'sweat' in the industry parlance). This monomer can be absorbed into the bulk PMMA and evaporate, while the lesscompletely degraded polymer is left as a particulate residue on the surface. In an effort to minimise this effect, the laser engraver system is forcefully vented (at about $150 \mathrm{cfm}$ ) by a pump.

The printer is designed to allow for successive patterns to be printed upwind, or downwind. In the former case, the residue of later chips is deposited on the earlier channels, leaving particulates in the channels. In the latter case the later chips are patterned onto a flat surface with particulates on it, possibly leaving an image in the surface below (after ablation). We chose to print going upwind and attempted to clean the chips after patterning (e.g. by sonication).

After these sections were completed, they were washed gently with a water-wetted sponge, dried and then annealed at $80 \mathrm{C}$ for an hour in a gravity convection oven (StabilTherm, Blue M Elec. Co.). They were then rinsed in ethanol (nominally 100\%), blown dry and assembled on a base holding two alignment pins. The assembly was sandwiched between two microscope slides top and bottom and clamped with standard stationary 'Bulldog' paper clips (Staples, Canada) and placed again in the oven for an hour at $110 \mathrm{C}$, and then allowed to 
cool to room temperature. The assembly now contains three bonded microfluidic chips that are cut free using the detachment power settings (for PMMA that is now $3 \mathrm{~mm}$ thick). The chips are then annealed again for an hour at $80 \mathrm{C}$ to relieve stresses. At various stages in their fabrication the chips were inspected by stereo microscope and camera (model zm-4tw3-for-9m, from AmScope, California, U.S.A.).

Following electrophoretic testing (described below), chips were cleaved apart into their component sections. By treating the channel as a scribe line, the chips can be cleaved reliably along the bottom of the channel in order to provide a measure of the depth uniformity of the laser fabrication.

Table 1. Settings for laser ablation.

\begin{tabular}{|l|l|l|l|l|}
\hline Mode & Power (\%) & Speed (\%) & PPI & \# of passes \\
\hline \hline Rastering of text & 36 & 100 & 500 & 1 \\
\hline Vector cut of frame & 20 & 5 & 1000 & 1 \\
\hline Vector cut of wells & 25 & 3 & 1000 & 1 \\
\hline Engraving of channels & 2.5 & 10 & 1000 & 2 \\
\hline Detachment of chips & 20 & 5 & 1000 & 1 \\
\hline
\end{tabular}

\subsection{Molecular Biology and Electrophoresis}

The chips were rinsed by applying suction and passing, in sequence, $\mathrm{mL}$ quantities of water, ethanol (nominally $100 \%$ ), water, $1 \mathrm{~N} \mathrm{HCl}$ and water in order to condition the chip. The chip was then dried and placed on a metal block that had been pre-heated to $50 \mathrm{C}$. A syringe was then used to fill the chip with a $2 \%$ agarose mixture in 1xTBE. Upon gelling, the agarose was cut out of the wells which were then filled with 1xTBE in three wells, and sample in the sample well. The sample was either a size standard or a combination of PCR products. The size standard was a 100 bp ladder (from New England Biolabs, NEB) with lengths of 100, 200, 300, 400, 500, $517,600,700,800,900,1000,1200$ and $1517 \mathrm{bp}$. With the resolution of our chip the 500 and 517 bands will run together, giving a total of 12 discernible bands. When using the size standard, we add $0.3 \mu L$ of sizer and $0.4 \mu L$ of $1 \mu M$ Sytox Orange (from Molecular Probes, Invitrogen) and $5.3 \mu L$ of $0.1 \mathrm{x}$ TBE, giving $15 \mathrm{ng}$ of DNA in the sample well with a ratio of 1:600 of dye to bp. When using the PCR products a mixture of $0.5 \mu L$ of each PCR products with $5.0 \mu L$ of $0.1 \mathrm{x}$ TBE was loaded. The PCR products were from the $\beta_{2} M$ gene (of $236 \mathrm{bp}$ ) and from the HV1 mitochondrial DNA D-loop region (345 bp). Because of the difficulty in reloading the chips they were only loaded with agarose once, and this typically allowed for many (e.g. 10) consecutive electrophoretic analyses.

The chips were loaded into a Microfluidics Toolkit (Micralyne, Edmonton, Canada), a system that applied high voltages and laser induced fluorescence (LIF) detection under computer control. The electrophoresis was performed according to the program described in Table 2 with the detection at $11 \mathrm{~mm}$, corresponding to an electric field of 42 and $25 \mathrm{~V} / \mathrm{cm}$ for injection and separation respectively. The LIF data was acquired at a frequency of $200 \mathrm{~Hz}$ and was subsequently analysed by a Python script that computed and plotted a running average (typically of a 10 sample neighbourhood). This averaging removed noise but did not affect resolution with these settings. As described below, the resolution obtained from agarose at these short distances is approximately $50 \mathrm{bp}$ and hence we expect to resolve 12 peaks from the DNA ladder.

From the electropherograms we estimated the resolution (R) in the region 200 to 300 bp by calculating:

$$
R=(300-200 b p) \frac{w_{1}+w_{2}}{t_{2}-t_{1}}
$$

where $w$ and $t$ represent the width and arrival times of the $200 \mathrm{bp}$ (peak 1) or $300 \mathrm{bp}$ (peak 2) peaks. 
Table 2. Settings for electrophoresis with the size standard with injection field of $42 \mathrm{~V} / \mathrm{cm}$, and a separation field of 25 $\mathrm{V} / \mathrm{cm}$. For the PCR products the durations were approximately halved (150s for the first injection, 11s subsequently). The term 'Float' indicates that the well was electrically disconnected.

\begin{tabular}{|l|l|l|l|l|l|}
\hline Step & Duration (s) & Buffer (V) & Buffer Waste(V) & Sample (V) & Sample Waste (V) \\
\hline \hline First injection & 250 & float & float & 0 & 70 \\
\hline First separation & 350 & 0 & 50 & float & float \\
\hline Following injections & 20 & float & float & 0 & 70 \\
\hline $\begin{array}{l}\text { Following separa- } \\
\text { tions }\end{array}$ & 350 & 0 & 50 & float & float \\
\hline
\end{tabular}

\section{RESULTS}

Although we were able to obtain electropherograms such as that of Fig. 2, we found the results to be erratic in terms of the resolution. We also found that we could only resolve the size standard up to peaks of a given length (a 'cut-off' length) and that this varied from chip to chip, as shown in Figures 3 to 6.

A clear pattern emerged in the printed arrays of chips - clean chips were ones that had no other chips upwind and these had a resolution of $50 \mathrm{bp}$ or so. Having a row of chips upwind degraded the resolution by about $15 \%$ and having two rows of chips upwind degraded the resolution by about $50 \%$. Further improvements in resolution may or may not be possible with this agarose concentration at these short separation lengths. Sonication was found to have little effect in removing the particulates.

We then cleaved the corresponding chips into their two sections of PMMA, and then longitudinally cleaved the section containing the channel. By inspecting the cleaved fragments of each channel and photographing them (in a side-on view), we were able to assemble the collage shown in Fig.7. Although only $3 \mathrm{~mm}$ of the separation channel is shown in each case, we were able to assemble a collage of the entire separation channel in each case (data not shown). From inspection it was clear that there was a strong correlation between smoothness of the channel bottom and the separation cut-off length. (The channel widths were fairly constant and varied by approximately 4\%.) Although developing a suitable metric for assessing the channel roughness was difficult, eventually we decided that a reasonable one was the number of times that the channel depth varied by more than $20 \%$, as shown in Table 3 .

With this metric in place we found that we could reliably build chips that could be used in a way representative of PCR-CE-based medical diagnostics, i.e. having adequate resolution in the range of $200-300 \mathrm{bp}$. As shown in Fig. 8, we can distinguish between two lengths of DNA in a manner much like that needed in a medical diagnostic based on PCR followed by electrophoretic sizing.

Table 3. PMMA channel quality vs. electrophoretic cut-off for the chips shown in Fig. 7.

\begin{tabular}{|l|l|l|}
\hline Chip Identity & \# Constrictions & Cut-off (bp) \\
\hline \hline $12-05-10-5 \mathrm{~A}$ & 2 & 1500 \\
\hline $12-06-27-3 \mathrm{~A}$ & 9 & 800 \\
\hline $12-06-27-7 \mathrm{~B}$ & 13 & 400 \\
\hline $12-07-04-5 \mathrm{~A}$ & 16 & 300 \\
\hline $12-06-27-4 \mathrm{C}$ & 17 & 300 \\
\hline
\end{tabular}

\section{DISCUSSION AND CONCLUSIONS}

As noted above, despite intense activity by companies such as Aclara and Caliper in developing techniques for performing electrophoresis in PMMA chips, this work has been primarily with embossing and injection moulding 
techniques. Most impressively, recent work by Liedert et al. (2012), ${ }^{4}$ shows how such chips could be mass manufactured with roll-to-roll embossing methods.

However, embossing and injection moulding are well-suited for mass manufacture, but are not well-adapted for rapid prototyping; $\mathrm{CO}_{2}$-laser-based fabrication thus has a considerable appeal for use in a rapid prototyping mode. Unfortunately, Locascio and others ${ }^{8,10}$ have suggested that laser processing can leave localised 'hot-spots' where the surface charge (and hence the zeta potential and EOF) vary.

Our initial experiments (e.g. with polymers such as POP7) showed erratic results and, generally, greatly degraded resolution. We assume that this is from such hot-spot-related effects. Although we were not able to directly measure the EOF, an indication of the magnitude of the EOF was that the $6 \mu L \mathrm{BW}$ well was empty (due to EOF) after several runs (and hence several minutes). Given that the channel cross section is approximately $100 \mu m$ on each side, this indicates an EOF of about $1 \mathrm{~mm} / \mathrm{s}$. The EOF mobility is given by the expression:

$$
\mu_{E O F}=\frac{-\epsilon \zeta}{\eta}=\frac{-\sigma_{e l} \lambda_{D}}{\eta}
$$

where $\epsilon$ is the permittivity of the medium, $\zeta$ the zeta potential of the surface, $\eta$ the viscosity of the medium, $\lambda_{D}$ the Debye length (a function of the charge concentration in the medium and $\sigma_{e l}$ is the surface charge density. (see e.g. Stone $/ 2004^{11}$ for more details).

Considering that the viscosity of POP7 is higher than that of water, and our fields are lower than those used by Sun et al., this suggests a zeta potential, surface charge and EOF mobility $\left(\mu_{E O F}\right)$ far higher than those seen by Sun et al.. ${ }^{7}$

Sun et al. extensively demonstrated their high-temperature bonding method of laser-cut PMMA and applied the resulting chips to the separation of DNA. Unfortunately they did not discuss the type of PMMA they used, nor the reproducibility of the results. However, with longer chips and a different seiving matrix (Hydroxypropylcellulose, HPC), they demonstrated the separation of a size ladder with what appears to be 5 or 10 bp resolution with channel depths and widths that varied by approximately $2 \%$. Using a current monitoring technique (e.g. ${ }^{12}$ ), their EOF was found to be $2.47 \times 10^{-4} \frac{\mathrm{cm}^{2}}{V \cdot s}$, a value that, as they noted, was comparable to that of pristine PMMA $\left(2.37 \times 10^{-4} \frac{\mathrm{cm}^{2}}{V \cdot s}\right)$. Although we attempted to determine the EOF in the same way we were unsuccessful, presumably because the surface charge of the laser-processed channel differed from that of the pristine cap. With the use of agarose though, these effects were largely or entirely suppressed.

With EOF variations suppressed by the use of agarose, we sought to understand the cut-off phenomenon. Surprisingly, Sun et al were able to obtain such good uniformity of depth and width (several times better than us) with what is essentially an earlier model of the same system that we are now using. Further investigation of our system revealed that the quality of the cut varied greatly with changes in driver software (e.g. whether the Universal Control Panel was updated) and application software (e.g. whether the CorelDraw application was allowed to update itself). To further complicate matters, the quality of the engraving is also degraded with smaller feature sizes, with long straight engraved channels being considerably smoother.

Much of this complexity stems from the fact that these $\mathrm{CO}_{2}$ systems are designed for applications where the cutting or engraving depth is not centrally important. The controlling software is designed to create a series of laser pulses to obtain the desired cut, perhaps with depth being a secondary consideration. Moreover, measuring the depth of such channels is significantly more difficult (requiring the longitudinal crack method) than measuring the width or length.

This work has shown how to control the surface charge variability that may be inherent in laser-processed PMMA. We have also developed methods and metrics to characterise channels and assess their suitability for the sizing of larger DNA. Future work will investigate the use of higher resolution separations while seeking to better control the channel roughness. Our present level of performance is suitable for medical diagnostics based on PCR followed by sizing, even in microchips that are the size of a postage stamp.

\section{ACKNOWLEDGMENTS}

We gratefully acknowledge the support of the Natural Sciences and Engineering Research Council of Canada, and that of Teledyne-DALSA. 


\section{REFERENCES}

1. Klank, H., Kutter, J. P., and Geschke, O. CO2-laser micromachining and back-end processing for rapid production of PMMA-based microfluidic systems. Lab On A Chip, 2(4):242-246, 2002.

2. Dubrow, R. and Caliper. Methods and compositions for performing molecular separations. US Patent, $\mathbf{6 , 0 4 2 , 7 1 0}(6,042,710): 21,2000$.

3. Tan, H. R., Sassi, A., and Cruzado, I. Dynamic coating with linear polymer mixture for electrophoresis. US Patent, 6787016 B2(6787016 B2):18, 2004.

4. Liedert, R., Amundsen, L. K., Hokkanen, A., Maki, M., Aittakorpi, A., Pakanen, M., Scherer, J. R., Mathies, R. A., Kurkinen, M., Uusitalo, S., Hakalahti, L., Nevanen, T. K., Siitari, H., and Soderlund, H. Disposable roll-to-roll hot embossed electrophoresis chip for detection of antibiotic resistance gene meca in bacteria. Lab On A Chip, 12(2):333-339, 2012. doi:10.1039/c1lc20782b.

5. Qi, H., Wang, X. S., Chen, T., Ma, X. M., and Zuo, T. C. Fabrication and characterization of a polymethyl methacrylate continuous-flow PCR microfluidic chip using CO2 laser ablation. Microsystem Technologiesmicro-and Nanosystems-information Storage and Processing Systems, 15(7):1027-1030, 2009.

6. Zhang, C. S. and Xing, D. Miniaturized PCR chips for nucleic acid amplification and analysis: latest advances and future trends. Nucleic Acids Research, 35(13):4223-4237, 2007.

7. Sun, Y., Kwok, Y. C., and Nguyen, N.-T. Low-pressure, high-temperature thermal bonding of polymeric microfluidic devices and their applications for electrophoretic separation. Journal of Micromechanics and Microengineering, 16(8):1681-1688, 2006. doi:10.1088/0960-1317/16/8/033.

8. Pugmire, D. L., Waddell, E. A., Haasch, R., Tarlov, M. J., and Locascio, E. Surface characterization of laserablated polymers used for microfluidics. Analytical Chemistry, 74(4):871-878, 2002. doi:10.1021/ac011026r.

9. Bowman, J. C. and Hammerlindl, A. Asymptote: A vector graphics language. The Communications of the TeX Users Group, 29:2:288-294, 2008.

10. Llopis, S. L., Osiri, J., and Soper, S. A. Surface modification of poly(methyl methacrylate) microfluidic devices for high-resolution separations of single-stranded DNA. Electrophoresis, 28(6):984-993, 2007. doi: 10.1002/elps.200600435.

11. Stone, H. A., Stroock, A. D., and Ajdari, A. Engineering flows in small devices: Microfluidics toward a lab-on-a-chip. Annual Review Of Fluid Mechanics, 36:381-411, 2004.

12. Huang, X. H., Gordon, M. J., and Zare, R. N. Current-monitoring method for measuring the electroosmotic flow-rate in capillary zone electrophoresis. Analytical Chemistry, 60(17):1837-1838, 1988. doi: 10.1021/ac00168a040. 


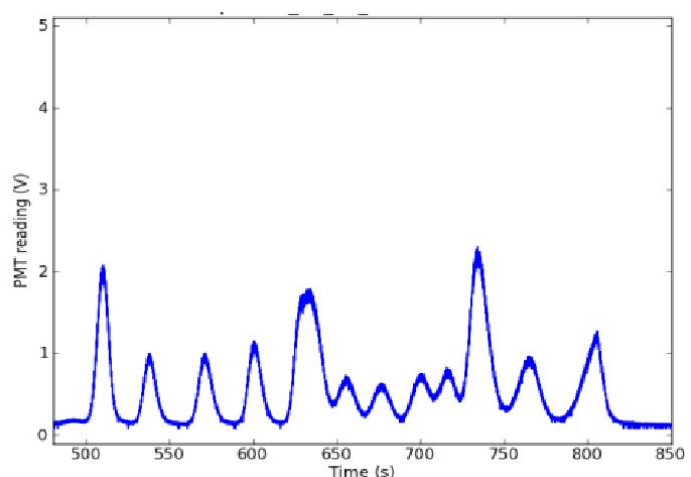

Figure 2. An electropherogram from chip 12-0510-5A, of the size standard from 100 to $1500 \mathrm{bp}$ with a resolution of about $50 \mathrm{bp}$ in the $100-200 \mathrm{bp}$ range. All 12 expected peaks are apparent.

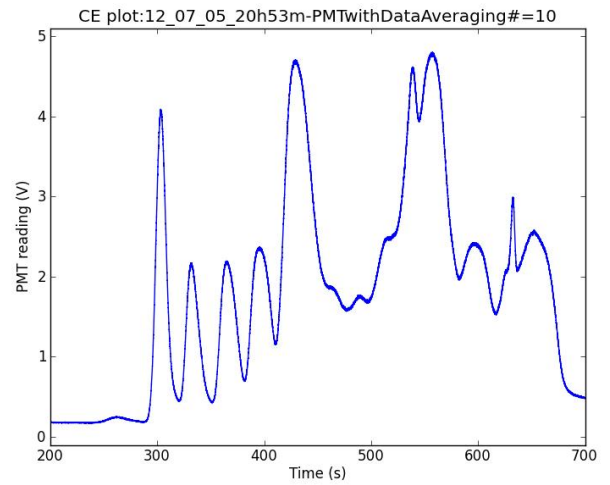

Figure 4. An electropherogram from chip 1206-27-7B, of the size standard (much as above). Only the first 4 peaks are clearly demarcated from their neighbours.

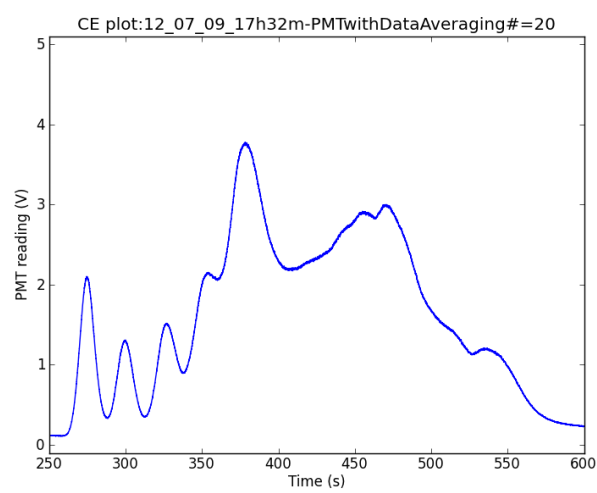

Figure 6. An electropherogram from chip 1207-04-5A, of the size standard (much as above). Only the first 3 peaks are clearly demarcated from their neighbours.

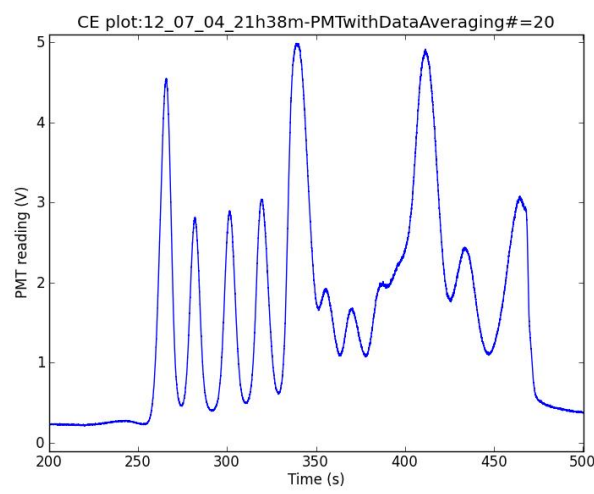

Figure 3. An electropherogram from chip 1206-27-3A, of the size standard (much as above). Only the first 5 peaks are clearly demarcated from their neighbours.

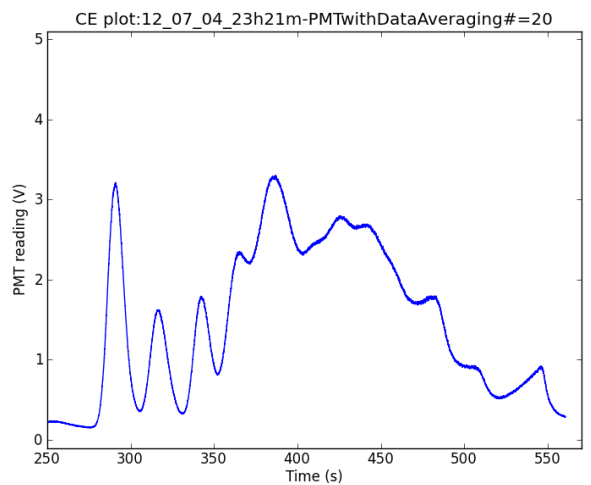

Figure 5. An electropherogram from chip 1206-27-4C, of the size standard (much as above). Only the first 3 peaks are clearly demarcated from their neighbours. 


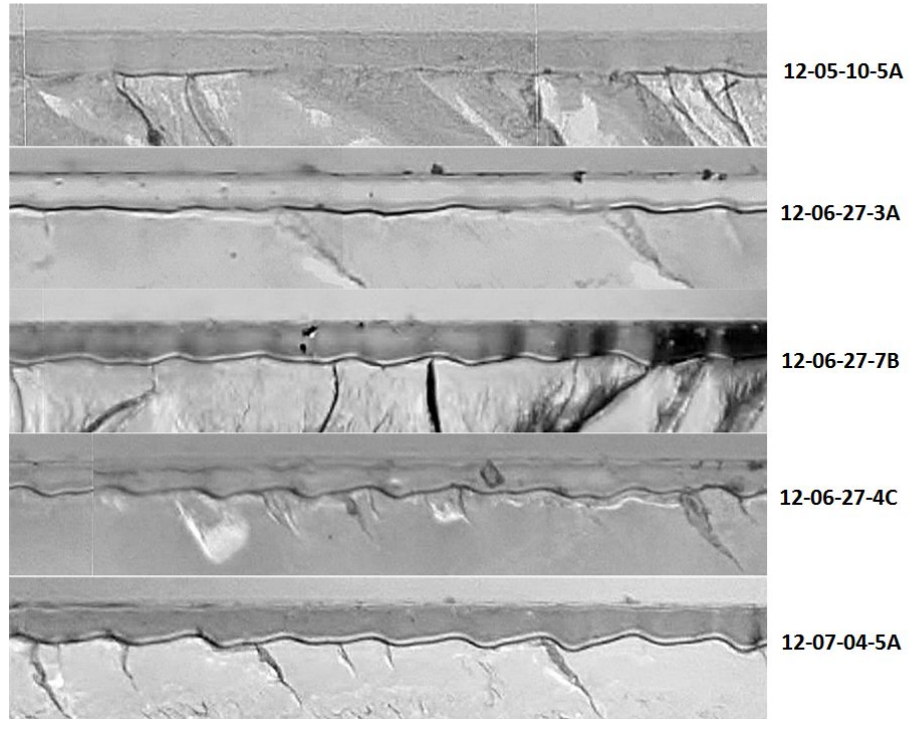

Figure 7. Collage showing depths of each of the channels for the chips corresponding to the above electropherograms. The regions shown are the last $3 \mathrm{~mm}$ of the separation channel up to $1.5 \mathrm{~mm}$ from the BW well but are representative of the separation channel as a whole.

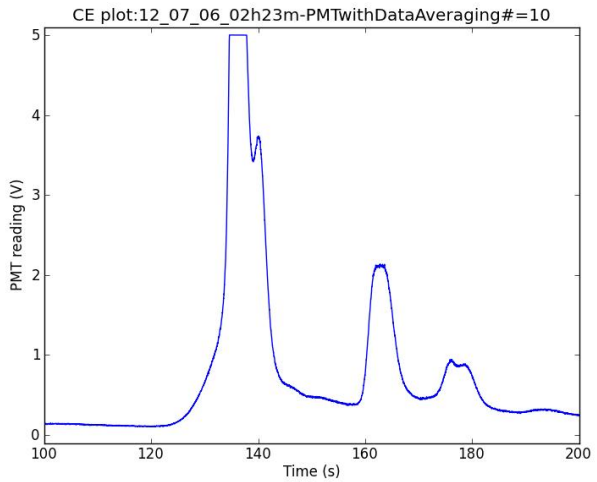

Figure 8. An electropherogram representative of a PCR-CE-based diagnostic, showing primers (near $140 \mathrm{~s}$ ) and a $\beta 2 M$ product (near $165 \mathrm{~s}$ ) and an HV1 product (near $175 \mathrm{~s}$ ), at 236 bp and $345 \mathrm{bp}$ respectively. 